\title{
Ultrahigh energy cosmic rays from dark matter annihilation
}

\author{
Rainer Dick ${ }^{\mathrm{a}}$, Pasquale Blasi ${ }^{\mathrm{b}}$ and Edward W. Kolb ${ }^{\mathrm{cd}}$ \\ ${ }^{\text {a}}$ Department of Physics and Engineering Physics, University of Saskatchewan, \\ 116 Science Place, Saskatoon, Canada SK S7N 5E2 \\ bINAF/Osservatorio Astrofisico di Arcetri, Largo E. Fermi 5, 50125 Firenze, Italy \\ ${ }^{\mathrm{c} N A S A}$ /Fermilab Astrophysics Center, Fermi National Accelerator Laboratory, \\ Batavia, Illinois 60510-0500 \\ ${ }^{\mathrm{d}}$ Department of Astronomy and Astrophysics, Enrico Fermi Institute, The University of Chicago, \\ Chicago, Illinois 60637-1433
}

Annihilation of clumped superheavy dark matter provides an interesting explanation for the origin of ultrahigh energy cosmic rays.

The predicted anisotropy signal provides a unique signature for this scenario.

\section{INTRODUCTION}

Explaining the origin of cosmic rays at energies above the Greisen-Zatsepin-Kuzmin bound $E_{G Z K} \simeq 4 \times 10^{19} \mathrm{eV}$ is an interesting and puzzling scientific challenge for several reasons:

AGASA observes a flux of order of one cosmic ray event per $100 \mathrm{~km}^{2}$ per year at energies above $10^{20} \mathrm{eV}[\mathrm{]}]$, thus confirming the flux seen by previous smaller ground based arrays on the northern hemisphere], see e.g. [2].

On the other hand, energy loss of electromagnetically interacting particles due to scattering with the CMB background indicates that these ultrahigh energy cosmic rays (UHECRs) should originate within a radius of order $100 \mathrm{Mpc}$ [4 6], and sufficiently powerful astrophysical acceleration mechanisms are not easy to find.

This has motivated proposals that superheavy dark matter may be the origin of ultrahigh energy

\footnotetext{
${ }^{1}$ But see [3] for a recent discussion of the pertinent problem to reconstruct primary cosmic ray energies from air showers. A new analysis presented there lowered the estimate on the energy of the highest energy event observed with the Haverah Park Array from above $10^{20} \mathrm{eV}$ to $8.3 \times 10^{19} \mathrm{eV}$ and also indicates a smaller flux at the high energy end of the spectrum. Of course, the very existence of UHECRs is not questioned by these findings.
}

cosmic rays:

This includes in particular Hill's proposal of monopolonium decay [7], the decay of superheavy dark matter relics proposed by Berezinsky et al. [8] and by Kuzmin and Rubakov [9], and most recently our proposal of collisional annihilation of superheavy dark matter [10,11]. While decay scenarios for UHECRs should imply domination of the galactic center in the distribution of arrival directions, the proposal of collisional annihilation predicts that with sufficient statistics from the upcoming Auger observatory a clumpy structure should emerge in the galactic source distribution: Unitarity bounds on reaction cross sections imply that a smooth superheavy dark matter distribution in the galactic halo would not yield the observed flux through collisional annihilation, but the overdensity in dark matter clumps in the halo may well account for the observed flux [10].

The common virtues of these top-down scenarios are:

- a natural explanation of the seemingly random distribution of arrival directions;

- avoidance of collisional damping and the GZK bound due to origination in the galactic halo;

- no need for an extremely powerful astrophysical acceleration mechanism. 
Very high-energetic neutrinos both from superheavy dark matter decay and from annihilation have very recently been discussed in [12, and some recent overviews of top-down scenarios for UHE cosmic rays can be found in 13 15].

Superheavy dark matter particles cannot arise as a consequence of standard thermal freeze out, but it has been pointed out that dark particles might have never experienced local chemical equilibrium during the evolution of the universe, and that their mass may be in the range $10^{12}$ to $10^{19} \mathrm{GeV}$, much larger than the mass of thermal WIMPS 16 19]. Since these WIMPS would be much more massive than thermal WIMPS, such superheavy dark particles have been called wIMPZILLAS [19].

WIMPZILLAS may be created during bubble collisions if inflation is completed through a firstorder phase transition [20.21; at the preheating stage after the end of inflation with masses easily up to the GUT scale 22] or even up to the Planck scale [23,24]; or during the reheating stage after inflation [18]. WIMPZILLAs may also be generated in the transition between an inflationary and a matter-dominated (or radiationdominated) universe due to the "nonadiabatic" expansion of the background spacetime acting on the vacuum quantum fluctuations [16,25,26]. The distinguishing feature of this mechanism is the capability of generating particles with mass of the order of the inflaton mass even when the particles only interact extremely weakly (or not at all) with other particles.

The mass $m$ of unstable dark matter which decays through gravitational couplings is constrained by the fact that the lifetime is only of order $\tau \leq m_{\text {Planck }}^{2} / m^{3}$ 27,28], and therefore unstable heavy relics must decay through strongly suppressed channels which effectively correspond to sub-gravitational couplings. This provided one motivation for the collisional annihilation proposal in [10], since the need for 2-particle collisions implies a larger lifetime of annihilating WIMPZILlAS as opposed to decaying superheavy particles.

\section{THE FLUX FROM ANNIHILATION IN DARK MATTER CLUMPS}

The spectral fluxes from decay or annihilation of WIMPZILLAS of mass $M_{X}$ and particle density $n_{X}(\mathbf{r})$ are

$\mathcal{F}_{d}(E)=\frac{d \mathcal{N}\left(E, M_{X} / 2\right)}{d E} \frac{1}{\tau_{d}} \int d^{3} \mathbf{r} \frac{n_{X}(\mathbf{r})}{2 \pi\left|\mathbf{r}_{\odot}-\mathbf{r}\right|^{2}}$

and

$\mathcal{F}(E)=\frac{d \mathcal{N}\left(E, M_{X}\right)}{d E}\left\langle\sigma_{A} v\right\rangle \int d^{3} \mathbf{r} \frac{n_{X}(\mathbf{r})^{2}}{2 \pi\left|\mathbf{r}_{\odot}-\mathbf{r}\right|^{2}},(2)$

respectively. The shape of the spectrum is determined by the function $d \mathcal{N}\left(E, E_{\text {jet }}\right) / d E$, which gives the number of sufficiently long-lived particles per energy interval emerging from a primary jet of energy $E_{\text {jet. }}$. Birkel and Sarkar and Berezinsky and Kachelriess have used Monte Carlo simulations to calculate the spectrum from decay of superheavy relics [29,30]. Fodor and Katz as well as Sarkar and Toldrà employed numerical integrations of the DGLAP evolution equations [31, 32]. The resulting spectrum in the interesting range between $10^{19} \mathrm{eV}$ and $10^{20} \mathrm{eV}$ is similar to the spectrum from the old modified leading log approximation (MLLA) [33], which was employed by Berezinsky et al. in their initial proposal of UHE cosmic rays from decay of superheavy dark matter decay [8].

We had also used the MLLA limiting spectrum in our proposal of WIMPZILLA annihilation 10 (Fig. 1).

The annihilation cross sections from the normalization in Fig. 11 were calculated for a smooth NFW [34 background halo. These cross sections would be in conflict with unitarity bounds on reaction cross sections for particles of mass $M_{X} \geq 10^{12} \mathrm{GeV}$ and therefore the flux from WIMPZILLA annihilation in the smooth halo component will (most likely) be negligible?. However, a fraction around a few percent of the dark matter

\footnotetext{
${ }^{2}$ There is also the possibility that WIMPZILLAS form metastable bound states, similar to monopolonium. Then the contribution from the smooth halo component could be important and would resemble the anisotropy signature expected from monopolonium or superheavy dark matter decay.
} 

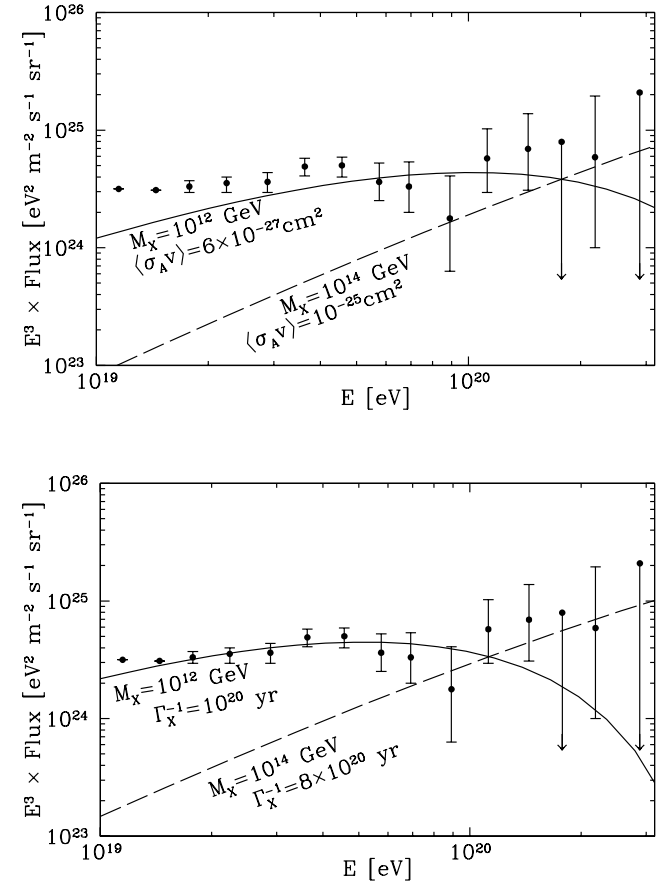

Figure 1. UHE cosmic ray spectra from superheavy particle annihilation (upper panel) or decay (lower panel).

in the halo is expected to exist in dense isothermal dark matter sub-clumps 3 , and due to large overdensities in the cores WIMPzILLA annihilation in these sub-clumps can comfortably generate the observed UHE flux with annihilation cross sections well below the unitarity bounds 10. This does not change the good fit of the annihilation model to the spectrum, which is a concequence of $d \mathcal{N}\left(E, M_{X}\right) / d E$, since the difference between events originating in the smooth and clumpy components affects only the overall factor $\int d^{3} \mathbf{r} n_{X}(\mathbf{r})^{2}\left|\mathbf{r}_{\odot}-\mathbf{r}\right|^{-2}$ in (2).

Due to the negligibility of a possible contribution from the smooth halo component a clumpy source structure will be an unmistakable signature of collisional WIMPZILLA annihilation as a

\footnotetext{
${ }^{3}$ See e.g. 355 for evidence for sub-clumps from $N$-body simulations, and 36] for evidence from gravitational lensing.
}

source of UHE cosmic rays. In particular, nearby sub-clumps will appear as hot spots in the sky (see Fig. 4 in Ref. [10]).

To calculate the expected overall anisotropy (after averaging over the clumps in the case of annihilation) one can integrate the local decay or annihilation rates entering in (11) and (2) along lines through the galactic halo, taking into account the galactic halo density and the expected sub-halo distribution, respectively.

The left panel in Fig. 2 shows the result for annihilation in a relatively broad subhalo distribution with the sub-clump density dropping off at a characteristic radius $R_{c}^{c l}=20 \mathrm{kpc}$ (see 10] for a discussion of the expected sub-halo distribution).
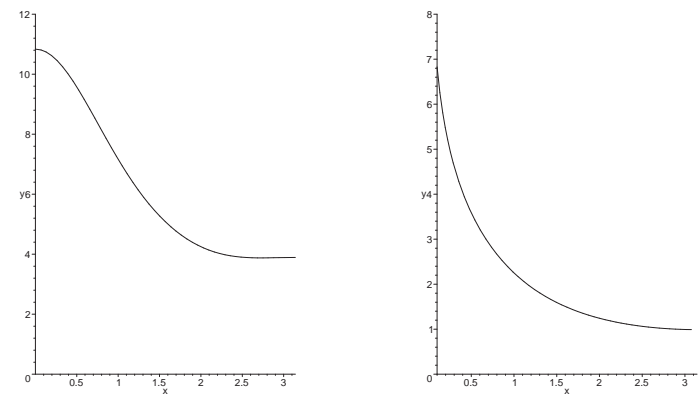

Figure 2. The left panel shows the envelope of the UHE flux from annihilation in a galactic subhalo distribution as a function of the angle towards the galactic center, whereas the right panel shows the UHE flux from decay in a galactic NFW profile.

\section{CONCLUSION}

All top-down scenarios for generation of UHECRs from superheavy dark matter predict a large photonic component in cosmic ray primaries above $E_{G Z K} \simeq 4 \times 10^{19} \mathrm{eV}$, and a more or less dominant role of the galactic center:

Both annihilation in bound states (monopolonium or "WIMPZILlONIUM" decay) and decay of superheavy relics imply that the dominant smooth dark matter halo distribution would be the primary source of UHECRs, whence the distribution of UHECR arrival directions should track the overall dark matter halo.

Collisional annihilation, on the other hand, would almost exclusively appear in the cores of 
local dark matter overdensities, and therefore would primarily track local overdensities. Here some domination of arrival directions pointing back towards the galactic center is expected because the sub-clump population is expected to drop off with distance from the galactic center. A nearby clump out of the line of sight to the galactic center could dominate the local signal and appear as a hot spot.

The southern site of the Auger observatory sees the galactic center, and therefore the prediction of a clumpy source distribution with some accumulation towards the galactic center should be testable after about three years of full operation of the southern site, which should yield of the order of 100 UHECR events with energies at and beyond $10^{20} \mathrm{eV}$.

\section{REFERENCES}

1. N. Sakaki et al. (AGASA Collaboration), in Proceedings of the 27th ICRC (Hamburg), Copernicus Gesellschaft, Hamburg 2001, pp. 333-336.

2. M. Nagano, A.A. Watson, Rev. Mod. Phys. 72 (2000) 689.

3. M. Ave, J. Knapp, J. Lloyd-Evans, M. Marchesini, A.A. Watson, astro-ph/0112253.

4. K. Greisen, Phys. Rev. Lett. 16 (1966) 748; G.T. Zatsepin, V.A. Kuzmin, JETP Lett. 4 (1966) 78 .

5. T. Stanev, R. Engel, A. Mücke, R.J. Protheroe, J.P. Rachen, Phys. Rev. D 62 (2000) 093005.

6. M. Blanton, P. Blasi, A. Olinto, Astropart. Phys. 15 (2001) 275.

7. C.T. Hill, Nucl. Phys. B 224 (1983) 469.

8. V. Berezinsky, M. Kachelrieß, A. Vilenkin, Phys. Rev. Lett. 79 (1997) 4302.

9. V.A. Kuzmin, V.A. Rubakov, Phys. Atom. Nucl. 61 (1998) 1028.

10. P. Blasi, R. Dick, E.W. Kolb, astroph/0105232, Astropart. Phys. (in press).

11. P. Blasi, R. Dick, E.W. Kolb, Nucl. Phys. B (Proc. Suppl.) 110 (2002) 494.

12. F. Halzen, D. Hooper, hep-ph/0110201, astro$\mathrm{ph} / 0204527$.

13. G. Domokos, S. Kovesi-Domokos, hep- ph/0107095.

14. P. Blasi, astro-ph/0110401.

15. S. Sarkar, hep-ph/0202013.

16. D.J.H. Chung, E.W. Kolb, A. Riotto, Phys. Rev. D 59 (1999) 023501.

17. D.J.H. Chung, E.W. Kolb, A. Riotto, Phys. Rev. Lett. 81 (1998) 4048.

18. D.J.H. Chung, E.W. Kolb, A. Riotto, Phys. Rev. D 60 (1999) 063504.

19. E.W. Kolb, D.J.H. Chung, A. Riotto, in Dark Matter in Astrophysics and Particle Physics 1998, IoP Publishing, Bristol 1999, pp. 592611.

20. J.D. Barrow, E.J. Copeland, E.W. Kolb, A.R. Liddle, Phys. Rev. D 43 (1991) 977.

21. A. Masiero, A. Riotto, Phys. Lett. B 289 (1992) 73.

22. E.W. Kolb, A. Riotto, I.I. Tkachev, Phys. Lett. B 423 (1998) 348.

23. D.J.H. Chung, E.W. Kolb, A. Riotto, I.I. Tkachev, Phys. Rev. D 62 (2000) 043508.

24. G.F. Giudice, M. Peloso, A. Riotto, I. Tkachev, JHEP 9908 (1999) 014.

25. V. Kuzmin, I. Tkachev, JETP Lett. 68 (1998) 271, Phys. Rev. D 59 (1999) 123006, Phys. Rep. 320 (1999) 199.

26. D.J.H. Chung, P. Crotty, E.W. Kolb, A. Riotto, Phys. Rev. D 64 (2001) 043503.

27. R. Dick, Fortschr. Phys. 45 (1997) 537.

28. R. Dick, Phys. Lett. B 510 (2001) 10.

29. M. Birkel, S. Sarkar, Astropart. Phys. 9 (1998) 297.

30. V. Berezinsky, M. Kachelriess, Phys. Rev. D 63 (2001) 034007.

31. Z. Fodor, S.D. Katz, Phys. Rev. Lett. 86 (2001) 3224.

32. S. Sarkar, R. Toldrà, Nucl. Phys. B 621 (2002) 495.

33. Ya.I. Azimov, Yu.L. Dokshitzer, V.A. Khoze, S.I. Troyan, Phys. Lett. B 165 (1985) 147, Z. Phys. C 27 (1985) 65, Z. Phys. C 31 (1986) 213.

34. J.F. Navarro, C.S. Frenk, S.D.M. White, Astrophys. J. 462 (1996) 563.

35. B. Moore, C. Calcáneo-Roldán, J. Stadel, T. Quinn, G. Lake, S. Ghigna, F. Governato, Phys. Rev. D 64 (2001) 063508.

36. N. Dalal, C.S. Kochanek, astro-ph/0111456. 